\title{
Further Expansion of the Mutational Spectrum of 3MC Syndrome: A Novel MASP1 Pathogenic Variant in a Male Patient
}

\author{
Nihat Bugra Agaoglu ${ }^{a, b}$ Ozlem Akgun Dogan ${ }^{a, c}$
}

${ }^{a}$ GLAB (Genomic Laboratory), Umraniye Training and Research Hospital, Istanbul, Turkey; bepartment of Medical Genetics, Umraniye Training and Research Hospital, Istanbul, Turkey; 'Department of Pediatric Genetics, Umraniye Training and Research Hospital, Istanbul, Turkey

\section{Established Facts}

- $3 \mathrm{MC}$ syndrome is a rare autosomal recessive syndrome characterized by facial dysmorphism, developmental and growth delay, and additional multisystemic findings.

- Biallelic pathogenic variants in MASP1, COLEC11, and COLEC10 are responsible for 3MC syndrome. To date, only 16 homozygous/compound heterozygous pathogenic variations (12 missense, 3 splice site, 1 deletion) in 27 patients from 22 families have been reported in MASP1-associated 3MC syndrome.

- Identifying new patients and reporting their clinical and molecular findings will enable delineating this rare syndrome's phenotypic and genotypic spectrum.

\section{Novel Insights}

- We report on a patient with a novel homozygous splice site mutation in MASP1.

- Pyloric stenosis and relative macrocephaly are findings previously described in only 2 patients, and their association with the syndrome had been reported controversial. The presence of these findings in our patient supports considering the abovementioned as components of $3 \mathrm{MC}$ syndrome.

\section{Keywords}

3MC syndrome $\cdot$ MASP1 - Dysmorphism - Novel variant .

Macrocephaly · Pyloric stenosis · Prenatal finding

\section{Abstract}

The $3 \mathrm{MC}$ syndrome is a rare autosomal recessive syndrome characterized by facial dysmorphism, multiple congenital abnormalities, and postnatal growth deficiency. Hypertelorism, blepharophimosis, blepharoptosis, high-arched eyebrows, and cleft lip/palate compose the facial gestalt, which is the key component for diagnosing the syndrome. Biallelic pathogenic variants in MASP1, COLEC11, and COLEC10 are responsible for $3 \mathrm{MC}$ syndrome in which both genotypic and phenotypic heterogeneity is described. To date, 16 homozygous/compound heterozygous pathogenic variations in 27 patients from 22 families have been reported in the MASP1 gene associated with 3MC syndrome. Here, we report a male patient with a novel homozygous pathogenic variant in MASP1 in whom macrocephaly, pyloric stenosis,
Correspondence to:

Nihat Bugra Agaoglu, nbagaoglu@ hotmail.com 
and prenatal findings including polyhydramnios, aortic dilatation, and intracranial cysts beside the distinctive facial features were detected. Reporting detailed clinical and molecular findings in patients is pivotal in terms of enabling the phenotypic and genotypic spectrum of this rare syndrome to be delineated.

(c) 2021 S. Karger AG, Basel

\section{Introduction}

The $3 \mathrm{MC}$ syndrome is a rare autosomal recessive syndrome characterized by facial dysmorphism, multiple congenital abnormalities, and postnatal growth deficiency. It is an umbrella diagnosis based on the initials of the Michels, Malpuech, Carnevale, and Mingarelli syndromes, which share overlapping phenotypic features, especially in the facial gestalt [Michels et al., 1978; Carnevale et al., 1989; Malpuech et al., 1983]. Hypertelorism, blepharophimosis, blepharoptosis, high-arched eyebrows, and cleft lip/palate are the main components of the dysmorphic features. Skeletal anomalies including craniosynostosis and radioulnar synostosis, anterior chamber defects of the eye, hearing loss, caudal appendage, umbilical hernia, and cardiac and urogenital anomalies are the other reported features of the syndrome [Sirmaci et al., 2010; Atik et al., 2015; Urquhart et al., 2016; Gardner et al., 2017; Munye et al., 2017; Basdemirci et al., 2019].

Biallelic pathogenic variants(PV) in MASP1, COLEC11, and COLEC10 are responsible for 3MC syndrome [Sirmaci et al., 2010; Graul-Neumann et al., 2018], and all are involved in the lectin complement pathway [Degn et al., 2011]. Among these, MASP1 has been shown to play an essential role in both craniofacial development and complement activation [Rooryck et al., 2011]. To date, 16 homozygous/compound heterozygous PV (12 missense, 3 splice-site, 1 deletion) in 27 patients from 22 families have been reported in the MASP1-associated 3MC syndrome [Sirmaci et al., 2010; Atik et al., 2015; Urquhart et al., 2016; Gardner et al., 2017; Munye et al., 2017; Graul-Neumann et al., 2018; Basdemirci et al., 2019].

Herein, we report on a patient with a novel homozygous splice-site pathogenic variant in MASP1 to further delineate both the phenotypic and genotypic spectrum of this rare syndrome.

\section{Case Report}

The proband is the fifth child of healthy, consanguineous (firstcousin marriage) Syrian parents. He was born via cesarean delivery at 35 weeks, with a birth weight of $2,420 \mathrm{~g}$ ( $<3 \mathrm{rd}$ centile). Birth length was $46 \mathrm{~cm}$ ( $3 \mathrm{rd}$ centile), and head circumference was 34.5 $\mathrm{cm}$ (25th-50th centile). In the prenatal period, polyhydramnios, intracranial cystic lesions, and dilatations in the descending aorta were detected in fetal ultrasonography; however, fetal MRI was not performed. During the neonatal period, he was hospitalized in the intensive care unit due to feeding difficulty, frequent vomiting, and pyloric stenosis. On the postnatal fourth day, surgical intervention was performed for gastric rupture. The patient was referred to the genetics department at the age of 8 months because of dysmorphic facial features. At admission, his weight was $6,000 \mathrm{~g}$ $(<3$ rd centile), length was $64 \mathrm{~cm}(<3$ rd centile), and his head circumference was $45 \mathrm{~cm}$ (15th centile). Physical examination revealed a high and wide forehead, large frontal fontanel, hypertelorism, blepharophimosis, blepharoptosis, epicanthus inversus, high-arched eyebrows, low-hanging columella, broad nasal bridge, retrognathia, skin tag at xiphoid process, umbilical hernia, clinodactyly of 5th fingers in both hands, hypoplastic scrotum, cryptorchidism, and a sacral dimple (Fig 1). The developmental milestones were compatible with his peers. Parents mentioned a sibling with similar facial features and cleft palate who died at the age of 3 months due to congenital heart disease.

The audiometric evaluation and eye examination were normal. Echocardiography revealed a small muscular ventricular septal defect, patent ductus arteriosus, and patent foramen ovale. Transfontanel ultrasonography was normal. Abdominal ultrasonography revealed lobulated contoured kidneys fused at the base at the midline pelvic region (horseshoe kidney). With the clinical findings and similarly affected sibling history, we considered 3MC syndrome in the patient. Clinical exome sequencing was performed since the genetic heterogeneity defined in the syndrome.

\section{Method and Results}

Automatic DNA isolation was performed by the standard protocols of the QIAAmp DNA Mini (Qiagen) kit from peripheral blood samples. The sequencing was done on Illumina NextSeq 500 platform within the test scope with SOPHIA Clinical Exome Solution using Illumina V2 chemicals. Sequence analysis covered each gene's coding region, including all coding exons \pm 10 bp of adjacent intronic sequences, and each nucleotide is read at a depth of at least $50 \times$. Any variants that fall outside these regions and exonic variants with a minor allele frequency of less than $10 \%$ were considered as false positives and not analyzed. The DNA sequences were aligned to the NCBI Build36 (hg18) version of the human genome. The Sophia-DDM-V5.2 bioinformatics analysis software performed variant calling and data analysis. The interpretation of the variants was performed according to the 2015 


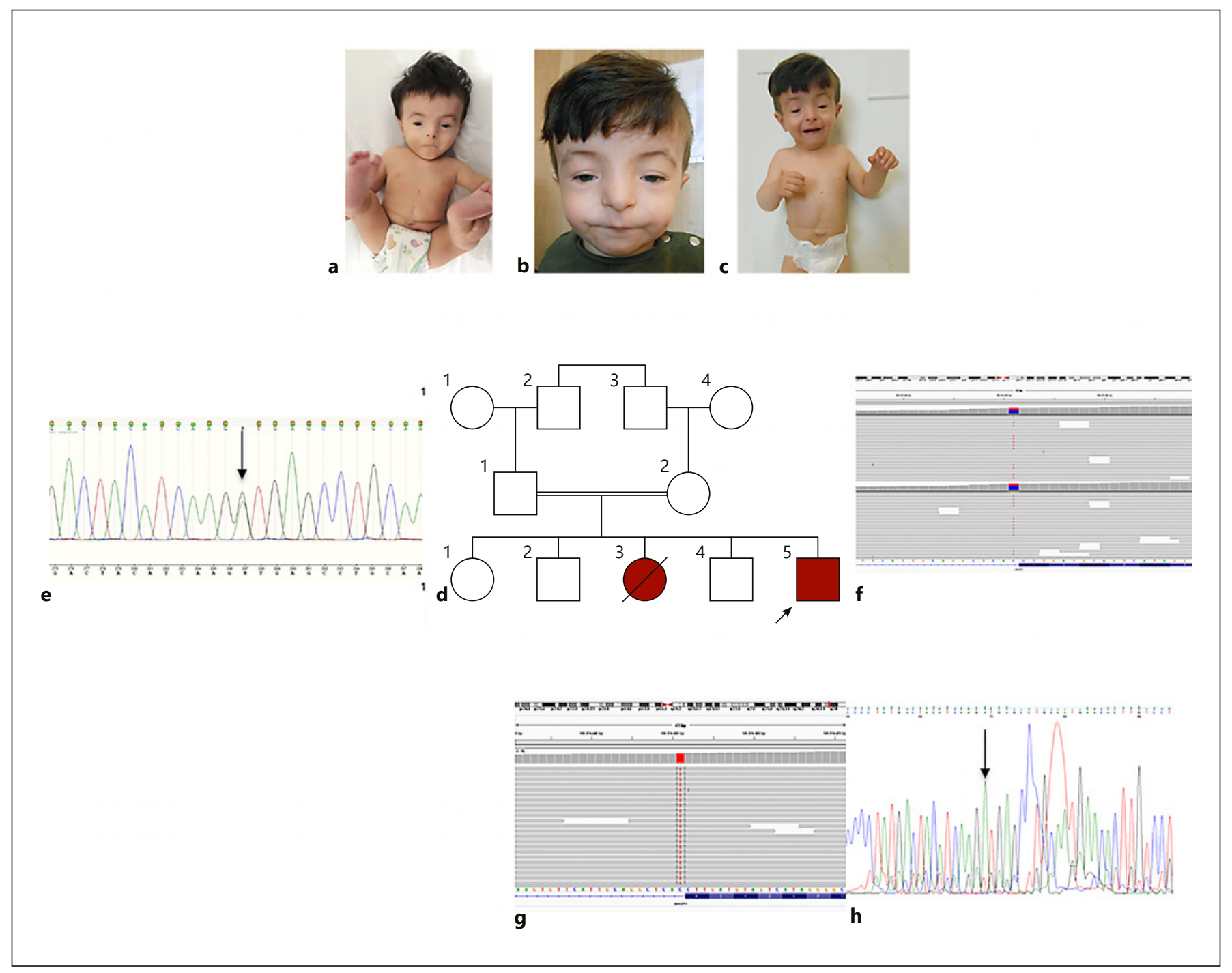

Fig. 1. Clinical picture of the patient at the age of 8 months (a) and 3 years and 6 months (b). Note the high and wide forehead, hypertelorism, blepharophimosis, blepharoptosis, epicanthus inversus, high-arched eyebrows, low-hanging columella, broad nasal bridge, retrognathia (a, b), skin tag at xiphoid process, and umbilical her- nia (a, c). Pedigree of the family (d). Sanger sequencing image of the MASP1 c.744+1G>A variant in the father (e) and Integrative Genomics Viewer (IGV) screenshot of the MASP1 c.744+1G>A variant in the mother $(\mathbf{f})$. IGV screenshot and Sanger sequencing images of the MASP1 c.744+1G>A variant in the patient $(\mathbf{g}, \mathbf{h})$.
ACMG standards and guidelines [Richards et al., 2015]. GnomAD, 1000 genome projects, dbSNP data were used as the control population. In silico prediction programs such as SIFT, PolyPhen, EIGEN, FATHMM-MKL, MutationTaster, and GERP were used for variant pathogenicity predictions.

In clinical exome sequencing analysis, MASP1 (NM_001031849), c.744+1G>A, homozygous splice donor variation was detected, which is a null variant the affecting donor splice site of MASP1 (PVS1). In silico prediction algorithms (EIGEN, FATHMM-MKL, and Muta-
tionTaster) indicate that the variant has a deleterious effect at protein level (PP3). The allele frequency of the variant is $2 / 251.232(0.00000796 \%)$, and it is not listed in a homozygous state in the current genome variant database gnomAD (PM2). Also, this variant has not been reported in ClinVar or in the Human Genome Mutation Database (HGMD). Considering the evidence described above (PVS1, PM2, PP3) and the clinical findings of the patient, the MASP1 (NM_001031849) c.744+1G>A variant has been classified as pathogenic. The variant was confirmed by Sanger sequencing. Primer sequences and 
Table 1. Summary of clinical findings in reported patients with $3 \mathrm{MC}$ syndrome and the present patient

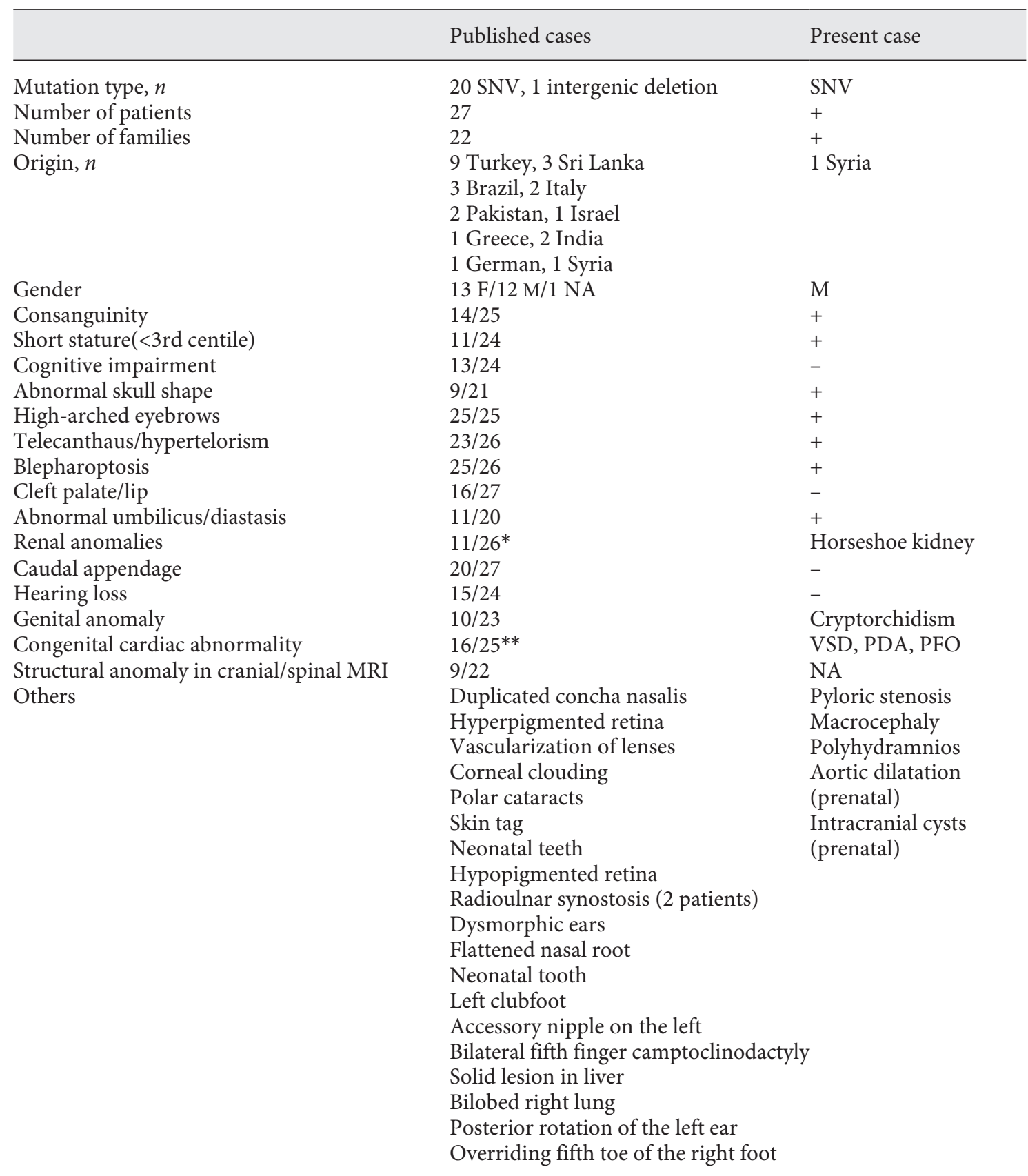

VSD, ventricular septal defect; PDA, patent ductus arteriosus; PFO, patent foramen ovale; NA, not available; +, present; -, absent.* Renal anomalies detected in previously reported patients: horseshoe kidney in 3 patients, cross renal ectopy in 2 patients, bilateral duplicated kidney, solitary ectopic kidney, left renal agenesis, and hydronephrosis in 1 patient each. ${ }^{* *}$ Congenital cardiac abnormalities detected in previously reported patients: patent ductus arteriosus in 9 patients, atrial septal defect in 7 patients, ventricular septal defect in 1 patient. In 5 patients, detailed information about cardiac abnormality could not be obtained. 


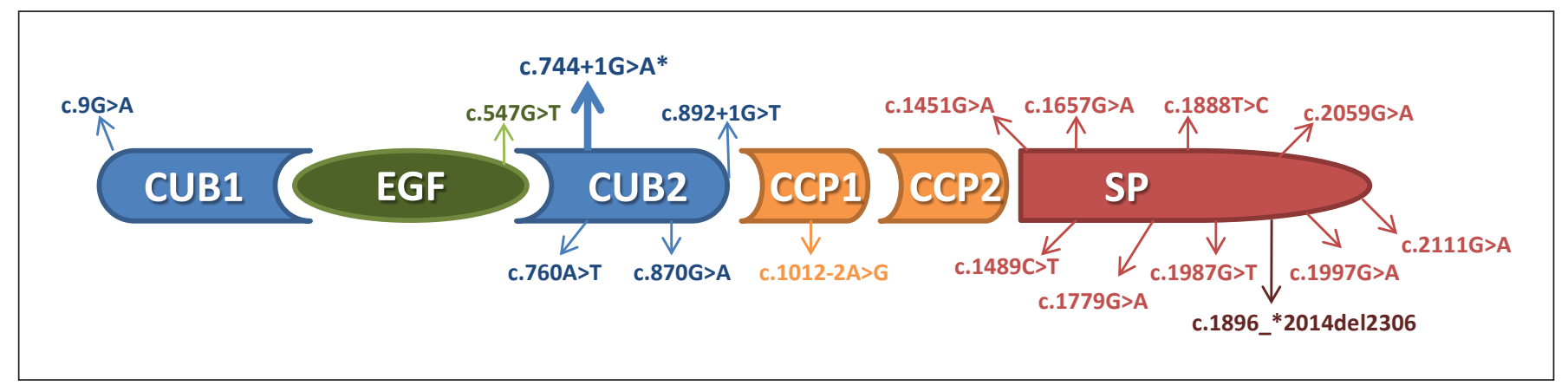

Fig. 2. Protein domains of MASP1 and distribution of reported pathogenic variants. The variant detected in the present case is indicated with *.

reaction conditions are available on request. In segregation analysis, the parents were found to be heterozygous for the same variation.

\section{Discussion and Conclusion}

The 3MC syndrome constitutes a highly heterogeneous group (both phenotype and genotype). So far, approximately 22 families have been reported with variants in MASP1 associated with $3 \mathrm{MC}$ syndrome [Basdemirci et al., 2019] (Table 1). All of the cases shared dysmorphic facial findings that were considered as pathognomonic, along with the rarer findings identified in other organ systems [Sirmaci et al., 2010; Atik et al., 2015; Urquhart et al., 2016; Gardner et al., 2017; Munye et al., 2017; Basdemirci et al., 2019; Garfield and Sergent, 2021]. Given both genotypic and phenotypic heterogeneity, describing new patients with novel clinical and molecular findings is essential to delineate phenotype-genotype correlation.

Herewith, we report on a male patient with a novel splice site variation in MASP1 with additional findings. Characteristic facial features, postnatal growth retardation, cardiac anomalies, horseshoe kidney, clinodactyly, skin tag at xiphoid process, and sacral dimple are among the previously reported findings which are also present in our patient. Among the facial features, cleft palate/lip is present in approximately $50 \%$ of the reported patients with 3MC syndrome, especially those with $\mathrm{PV}$ in MASP1[Atik et al., 2015]. Cleft lip/palate was not present in our patient; however, there is a history of a sibling with similar dysmorphic features and cleft palate resembling $3 \mathrm{MC}$ syndrome. Although segregation analysis could not be performed on the deceased sibling, this finding may suggest variable expressivity in MASP1, even in the same

3MC Syndrome: A Novel MASP1

Pathogenic Variant family. Intellectual disability/developmental delay (ID/ $\mathrm{DD}$ ) is considered as a component of the syndrome; however, the incidence is variable according to the underlying molecular etiology. ID/DD was reported slightly lower (28.5\%) among patients with MASP1 PV [Atik et al., 2015]. As compatible with this observation, our patient's developmental milestones were also consistent with his peers, but given the patient's early age, long-term followup is required to make a clear psychometric assessment.

Macrocephaly and pyloric stenosis reported in our patient are extremely rare findings among patients with $3 \mathrm{MC}$ syndrome. Macrocephaly was previously described in a patient by Graul-Neumann et al. [2018]; however, it was assessed as a familial finding, not a feature of the syndrome. In our patient, the parents' head circumference was within normal limits. Considering the patient reported by Graul-Neumannet al. [2018], we suggest that the relative macrocephaly is one of the rare findings of $3 \mathrm{MC}$ syndrome. Pyloric stenosis was previously reported in a patient with COLEC11 PV, and our patient is the first individual to have pyloric stenosis reported in MASP1 PV [Urquhart et al., 2016]. On the other hand, pyloric stenosis is considered a birth defect with multifactorial inheritance, especially when it does not accompany another anomaly. The incidence is 2.4 per 1,000 live births in Caucasians, and the male gender is frequently affected [Garfield and Sergent, 2021]. Therefore, it should be kept in mind that pyloric stenosis may be a secondary accompanying finding in the reported patients. Additional patients are needed to enroll this finding as a component of the syndrome.

Prenatal findings, including polyhydramnios, aortic dilatation, and intracranial cysts, are the novel features in our patient. Despite the approximately 22 reported families with MASP1-related 3MC syndrome so far, informa- 
tion on prenatal findings of the syndrome is scant. Recently, Lawson et al. [2020] reported an affected fetus with cleft palate, cardiac anomalies, and caudal appendage. In our patient, intracranial cysts were regressed after delivery; also, findings related to aortic dilatation were not detected in echocardiography at the age of 1 month. Since polyhydramnios may have been seen secondary to pyloric stenosis, and aortic dilatation or intracranial cysts could not be detected in the neonatal period, it is controversial to suggest that these findings are related to the syndrome [Pappalardo et al., 2009]. New patients are required to determine the association of these findings with the syndrome.

The MASP1 gene encodes 3 isoforms, MASP1, MASP3, and MASP4, which differ in their carboxyl terminals [Sirmaci et al., 2010; Atik et al., 2015]. MASP1 and MASP3 isoforms consist of 6 protein domains, while MASP4 has 4 protein domains [Sirmaci et al., 2010; Atik et al., 2015] (Fig. 2). Among these, the serine protease domain stands out as the most frequently affected domain in patients with 3MC syndrome and is present only in MASP1 and MASP3 isoforms [Sirmaci et al., 2010; Atik et al., 2015]. To date, there were $16 \mathrm{PV}$ in MASP1 associated with 3MC syndrome in the HGMD, including missense, nonsense variants, large deletion, and intronic variants that affect splice sites as in our patient. All reported so far in MASP1 are shown in Figure 2. The present MASP1 (NM_001031849), c.744+1G >A variant lies in the donor splice site affecting exon 5 and shared by all 3 isoforms. It is also found to be in a highly conserved region with PhyloP and GERP scores of 4.88 and 5.53, respectively. This variant is predicted to affect the CUB2 domain, the second most common protein domain in which PV are reported in 3MC syndrome (Fig 2). Among the patients with PV affecting the CUB2 domain, we figured out that in all but one patient had renal anomalies in addition to the characteristic features of the syndrome [Sirmaci et al., 2010; Urquhart et al., 2016] whereas, only one patient had renal anomaly among patients with PV in the serine protease domain [Sirmaci et al., 2010; Atik et al., 2015;Urquhart et al., 2016; Graul-Neumann et al., 2018; Basdemirci et al., 2019]. Our patient also had a horseshoe kidney, consistent with these patients' findings. However, the number of patients was insufficient to establish a reliable correlation between the renal features and affected protein domains.

In conclusion, we report on a male patient with a novel homozygous variant in MASP1 to expand the mutational spectrum of $M A S P 1$-related $3 \mathrm{MC}$ syndrome. Since there is a limited number of reported patients, identifying new patients and elaborating their clinical findings will increase clinicians' awareness and enable establishing phenotype-genotype correlations.

\section{Statement of Ethics}

This study was performed in accordance with the Declaration of Helsinki Principles. Written informed consent was obtained from the patients' parents for publication of this case report and any accompanying images.

\section{Conflict of Interest Statement}

The authors have no conflicts of interest to declare.

\section{Funding Sources}

The authors received no specific funding for this work.

\section{Author Contributions}

The authors contributed to the conception and design of the work, drafted and revised it critically, and approved the final version of the manuscript. The authors agree to be accountable for all aspects of the work in ensuring that questions related to the accuracy or integrity of any part of the work are properly investigated and resolved.

References

Atik T, Koparir A, Bademci G, Foster J 2nd, Altunoglu U, Mutlu GY, et al. Novel MASP1 mutations are associated with an expanded phenotype in $3 \mathrm{MC1}$ syndrome. Orphanet J Rare Dis. 2015;10:128.

Basdemirci M, Sen A, Ceylaner S. Novel mutation in MASP1 gene in a new family with $3 \mathrm{MC}$ syndrome. Clin Dysmorphol. 2019;28(2):913.

Carnevale F, Krajewska G, Fischetto R, Greco MG, Bonvino A. Ptosis of eyelids, strabismus, diastasis recti, hip defect, cryptorchidism, and developmental delay in two sibs. Am J Med Genet. 1989;33(2):186-9.

Degn SE, Jensenius JC, Thiel S. Disease-causing mutations in genes of the complement system. Am J Hum Genet. 2011;88(6):689-705.

Gardner OK, Haynes K, Schweitzer D, Johns A, Magee WP, Urata MM, et al. Familial Recurrence of 3MC Syndrome in Consanguineous Families: A Clinical and Molecular Diagnostic Approach With Review of the Literature. Cleft Palate Craniofac J. 2017;54(6):739-48.

Garfield K, Sergent SR. Pyloric Stenosis, in StatPearls [Internet]. Treasure Island. StatPearls Publishing; 2021.
Mol Syndromol 2021;12:379-385 DOI: $10.1159 / 000517370$
Agaoglu/Akgun Dogan 
Graul-Neumann LM, Mensah MA, Klopocki E, Uebe S, Ekici AB, Thiel CT, et al. Biallelic intragenic deletion in MASP1 in an adult female with 3MC syndrome. Eur J Med Genet. 2018; 61(7):363-8.

Lawson C, Blakemore KJ, Ryan R, Hooper JE, Tsimis M, Jelin A. Sacral protuberance with cleft lip and palate: Prenatal presentation of $3 \mathrm{MC}$ syndrome. Am J Med Genet A. 2020;182(7): $1812-4$.

Malpuech G, Demeocq F, Palcoux JB, Vanlieferinghen P. A previously undescribed autosomal recessive multiple congenital anomalies/ mental retardation (MCA/MR) syndrome with growth failure, lip/palate cleft(s), and urogenital anomalies. Am J Med Genet. 1983; 16(4):475-80.
Michels VV, Hittner HM, Beaud, et al. A clefting syndrome with ocular anterior chamber defect and lid anomalies. J Pediatr. 1978;93(3): 444-6.

Munye MM, Diaz-Font A, Ocaka L, Henriksen ML, Lees M, Brady A, et al. COLEC10 is mutated in $3 \mathrm{MC}$ patients and regulates early craniofacial development. PLoS Genet. 2017; 13(3):e1006679.

Pappalardo EM, Militello M, Rapisarda G, Imbruglia L, Recupero S, Ermito S, et al. Fetal intracranial cysts: prenatal diagnosis and outcome. J Prenat Med. 2009;3(2):28-30.

Richards S, Aziz N, Bale S, Bick D, Das S, GastierFoster J, et al. Standards and guidelines for the interpretation of sequence variants: a joint consensus recommendation of the American College of Medical Genetics and Genomics and the Association for Molecular Pathology. Genet Med. 2015;17(5):405-24.
Rooryck C, Diaz-Font A, Osborn DP, Chabchoub E, Hernandez-Hernandez V, Shamseldin $\mathrm{H}$, et al. Mutations in lectin complement pathway genes COLEC11 and MASP1 cause 3MC syndrome. Nat Genet. 2011;43(3):197-203.

Sirmaci A, Walsh T, Akay H, Spiliopoulos M Sakalar YB, Hasanefendioğlu-Bayrak A, et al. MASP1 mutations in patients with facial, umbilical, coccygeal, and auditory findings of Carnevale, Malpuech, OSA, and Michels syndromes. Am J Hum Genet. 2010;87(5):67986.

Urquhart J, Roberts R, de Silva D, Shalev S, Chervinsky E, Nampoothiri S, et al. Exploring the genetic basis of $3 \mathrm{MC}$ syndrome: Findings in 12 further families. Am J Med Genet A. 2016;170A(5):1216-24. 